\title{
Numerical Method for Three-Parameter Eigenvalue Problems using Newton's method based on Trace Theorem
}

\author{
Songita Boruah \\ Research scholar \\ Dept. Of Mathematics D.U \\ Dibrugarh Assam, India
}

\begin{abstract}
In this paper Newton's method using Trace Theorem for three-parameter eigenvalue problems are discussed and some numerical results are presented to illustrate the performance and application of the method
\end{abstract}

\section{General Terms}

Eigenvalue, Eigenvector, Multiparameter

\section{Key words}

Multiparameter, eigenvalue, eigenvector, Newton's method, Trace theorem

\section{INTRODUCTION}

Multiparameter eigenvalue problems are generalization of one-parameter eigenvalue problems and can be found when the method of separation of variables is applied to certain boundary value problems associated with partial differential equations. Typical examples are provided, for example by a vibrating membrane Roach [16] and a dynamical problem of homogeneous beam loaded by a vertical load Collatz [7].

Although the literature on multiparameter problems is over a century old, the attention of the mathematicians towards multiparameter problems started with the unifying work of Atkinson[1]. Much more works have been done in the field of one-parameter eigenvalue problems, both theoretically and numerically compared to two-parameter or more than two-parameter eigenvalue problems. Some works have been done theoretically in the field of multiparameter eigenvalue problems. Few authors namely Fox et. al[9], Sleeman[17], Baruah[3], Konwar[13], have dealt with the multiparameter eigenvalue problems numerically mainly in two parametric cases. Numerical methods applied to a three-parameter problems are very limited and hence some contribution in this area are always in needed.

\subsection{Three-Parameter Eigenvalue Problem} And Its Reduction To A System Of

\section{One-Parameter Problems}

A three-parameter eigenvalue problems in matrix form is as follows

$W_{1}(\lambda) \mathrm{x}=A_{10} x-\lambda_{1} A_{11} x-\lambda_{2} A_{12} x-\lambda_{3} A_{13} x=0$

$W_{2}(\lambda) \mathrm{y}=A_{20} y-\lambda_{1} A_{21} y-\lambda_{2} A_{22} y-\lambda_{3} A_{23} y=0$

$W_{3}(\lambda) \mathrm{z}=A_{30} z-\lambda_{1} A_{31} z-\lambda_{2} A_{32} z-\lambda_{3} A_{33} z=0$

Where $\lambda_{i} \in \mathfrak{£}, \mathrm{i}=1,2,3$ and

\author{
Arun Kumar Baruah \\ Professor \\ Dept. Of Mathematics D.U \\ Dibrugarh Assam, India
}

$$
\begin{aligned}
& x \in \mathfrak{£}^{n} \backslash\{0\}, \mathrm{A}_{10}, \mathrm{~A}_{11}, \mathrm{~A}_{12}, \mathrm{~A}_{13} \in \mathfrak{£}^{n \times n} \\
& y \in \mathfrak{£}^{m} \backslash\{0\}, \mathrm{A}_{20}, \mathrm{~A}_{21}, \mathrm{~A}_{22}, \mathrm{~A}_{23} \in \mathfrak{£}^{m \times m} \\
& z \in £^{p} \backslash\{0\}, \mathrm{A}_{30}, \mathrm{~A}_{31}, \mathrm{~A}_{32}, \mathrm{~A}_{33} \in £^{p \times p}
\end{aligned}
$$

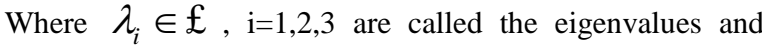
$x, y, z$ are called eigenvectors of the problem.

Problem(1.1.1) can be reduced to a system of three one-

parameter problems:

$$
\begin{aligned}
& \Delta_{1} u=\lambda_{1} \Delta_{0} u \\
& \Delta_{2} u=\lambda_{2} \Delta_{0} u \\
& \Delta_{3} u=\lambda_{3} \Delta_{0} u
\end{aligned}
$$

where $\Delta_{0}, \Delta_{1}, \Delta_{2}, \Delta_{3}$ are $(\mathrm{mnp}) \times(\mathrm{mnp})$ dimensional matrices defined as

$$
\begin{aligned}
& \Delta_{0}=A_{11} \otimes A_{22} \otimes A_{33}-A_{11} \otimes A_{23} \otimes A_{32} \\
& +A_{12} \otimes A_{23} \otimes A_{31}-A_{12} \otimes A_{21} \otimes A_{33} \\
& +A_{13} \otimes A_{21} \otimes A_{32}-A_{13} \otimes A_{22} \otimes A_{31} \\
& \Delta_{1}=A_{10} \otimes A_{22} \otimes A_{33}-A_{10} \otimes A_{23} \otimes A_{32} \\
& +A_{12} \otimes A_{23} \otimes A_{30}-A_{12} \otimes A_{20} \otimes A_{33} \\
& +A_{13} \otimes A_{20} \otimes A_{32}-A_{13} \otimes A_{22} \otimes A_{30} \\
& \Delta_{2}=A_{11} \otimes A_{20} \otimes A_{33}-A_{11} \otimes A_{23} \otimes A_{30} \\
& +A_{10} \otimes A_{23} \otimes A_{31}-A_{10} \otimes A_{21} \otimes A_{33} \\
& +A_{13} \otimes A_{21} \otimes A_{30}-A_{13} \otimes A_{20} \otimes A_{31} \\
& \Delta_{3}=A_{11} \otimes A_{22} \otimes A_{30}-A_{11} \otimes A_{20} \otimes A_{32} \\
& +A_{12} \otimes A_{20} \otimes A_{31}-A_{12} \otimes A_{21} \otimes A_{30} \\
& +A_{10} \otimes A_{21} \otimes A_{32}-A_{10} \otimes A_{22} \otimes A_{31} \\
& \text { And } \\
& u=x \otimes y \otimes z
\end{aligned}
$$

With $\otimes$ denoting the Kronecker product (or Tensor product) of two matrices discussed in (1.3). 
Theorem : Let $\left(\lambda_{1}, \lambda_{2}, \lambda_{3}\right)$ be an eigenvalue and $(\mathrm{x}, \mathrm{y}, \mathrm{z})$ a corresponding eigenvector of the system (1.1.1) then $\left(\lambda_{1}, \lambda_{2}, \lambda_{3}\right)$ is an eigenvalue of the system (1.1.2) and $u=x \otimes y \otimes z \quad$ is the corresponding eigenvector.

\subsection{The Kronecker Product}

Definition1.2. 1: The Kronecker product

$(. \otimes):. £^{m \times n} \times £^{p \times q} \rightarrow £^{m p \times n q}$ is defined by

$A \otimes B=\left[\begin{array}{rrr}a_{11} B & \mathrm{~L} & a_{1 n} B \\ \mathrm{M} & \mathrm{O} & \mathrm{M} \\ a_{n 1} B & \mathrm{~L} & a_{n n} B\end{array}\right]$

Where we use the standard notation $(A)_{i j}=a_{i j}$

The Kronecker product is a special case of the tensor product, and as such it inherits the properties of bilinearity and associativity, i.e.

$(k A) \otimes B=A \otimes(k B)=k(A \otimes B)$

$\mathrm{A} \otimes(\mathrm{B}+\mathrm{C})=\mathrm{A} \otimes \mathrm{B}+\mathrm{A} \otimes C$

$(\mathrm{A}+\mathrm{B}) \otimes \mathrm{C}=\mathrm{A} \otimes C+B \otimes C$

We now establish a famous property of the Kronecker product, from [9].

Lemma (Mixed product property). Let $A \in £^{m \times n}, B \in £^{p \times q}, C \in £^{n \times k}, D \in £^{q \times r}$. Then $(A \otimes B)(\mathrm{C} \otimes \mathrm{D})=(\mathrm{AC} \otimes \mathrm{BD})$.

In particular, if $A, B \in £^{m \times m}$ and $\mathrm{x}, \mathrm{y} \in £^{m}$ then $(A \otimes B)(\mathrm{x} \otimes \mathrm{y})=\mathrm{Ax} \otimes \mathrm{By}$.

\section{NEWTON METHOD USIN TRACE THEOREM}

Let $f(\lambda)=\left(\begin{array}{l}\operatorname{det} W_{1}(\lambda) \\ \operatorname{det} W_{2}(\lambda) \\ \operatorname{det} W_{3}(\lambda)\end{array}\right)=\left(\begin{array}{l}f_{1}(\lambda) \\ f_{2}(\lambda) \\ f_{3}(\lambda)\end{array}\right)$

Obviously, $\lambda \in i^{3}$ is an eigenvalue of the threeparameter eigenvalue problems (1.2.1) if and only if

$$
f(\lambda)=0
$$

In order to apply Newton's method to solve the nonlinear system (2.2), we need the partial derivatives of $f(\mathrm{c})$ with respect to the $\lambda_{1}, \lambda_{2}, \lambda_{3}$. To calculate these derivatives we employ the following Trace Theorem[].

Theorem: If the elements of the square matrix $\mathrm{B}(\mathrm{c})$ are differentiable functions of $\mathrm{c}$, then for any $\mathrm{c}$, we have for the derivative of the determinant det $\mathrm{B}(\mathrm{c})$ of $\mathrm{B}(\mathrm{c})$ :

$$
\frac{d(\operatorname{det} \mathrm{B}(\mathrm{c}))}{d c}=\operatorname{tr}\left[\operatorname{adjB}(\mathrm{c}) \cdot \frac{d B(\mathrm{c})}{d c}\right]
$$

And if $\operatorname{det} B(c)$ does not vanish $\frac{d(\operatorname{det} \mathrm{B}(\mathrm{c}))}{d c}=\operatorname{det} B(\mathrm{c}) \cdot \operatorname{tr}\left[\mathrm{B}^{-1}(\mathrm{c}) \cdot \frac{d B(\mathrm{c})}{d c}\right]$

Where tr and adj mean trace and adjoint, respectively and $\frac{d(\operatorname{det} \mathrm{B}(\mathrm{c}))}{d c}=\frac{d b_{i j}(\mathrm{c})}{d c}$

Using trace theorem, we have

$\frac{\partial\left(\mathrm{f}_{i}(\lambda)\right)}{\partial \lambda_{j}}=\frac{\partial\left(\operatorname{det}_{i}(\lambda)\right)}{\partial \lambda_{j}}=\operatorname{tr}\left[\operatorname{adj}\left(\mathrm{W}_{i}(\mathrm{c})\right) \cdot \frac{\partial W_{i}(\mathrm{c})}{\partial \lambda_{j}}\right]$

Then the $3 \times 3$ Jacobian $J_{f}(\lambda)$ of the function $f(\lambda)$ is

$$
\mathrm{J}_{f}(\lambda)=\operatorname{tr}\left[\operatorname{adj} \mathrm{W}_{i}(\mathrm{c}) \cdot \frac{\partial W_{i}(\mathrm{c})}{\partial \lambda_{j}}\right]
$$

If det $W_{i}(\mathrm{c}) \neq 0$, then

$$
\begin{aligned}
& J_{f}(\lambda)=\operatorname{det}_{i}(\mathrm{c}) \operatorname{tr}\left[\mathrm{W}_{i}^{-1}(\mathrm{c}) \frac{\partial W_{i}(\mathrm{c})}{\partial \lambda_{j}}\right] \\
& J_{f}(\lambda)=\operatorname{diag}\left(\operatorname{det} \mathrm{W}_{1}(\lambda), \operatorname{det}_{2}(\lambda), \operatorname{det} \mathrm{W}_{3}(\lambda)\right) \mathrm{H}(\lambda)
\end{aligned}
$$

Where $H(\lambda)=\operatorname{tr}\left[W_{i}^{-1}(\lambda) \frac{\partial W_{i}(\lambda)}{\partial \lambda_{j}}\right]$

Newton's method to approach a solution of (2.2) has the form

$$
\lambda^{(\mathrm{n}+1)}=\lambda^{(\mathrm{n})}-J_{f}^{-1}\left(\lambda^{(\mathrm{n})}\right) \mathrm{f}\left(\lambda^{(\mathrm{n})}\right), \mathrm{n}=0,1,2, \ldots \ldots
$$

Where $\lambda^{(0)}=\left(\lambda_{1}{ }^{(0)}, \lambda_{2}{ }^{(0)}, \lambda_{3}{ }^{(0)}\right)^{T}$ is an initial approximation.

\section{NUMERICAL EXAMPLE:}

We now present a numerical example to show the behaviour and application of our method

Consider the following system of equations

$$
\begin{aligned}
& \left(\begin{array}{ll}
1 & 0 \\
0 & 2
\end{array}\right)\left(\begin{array}{l}
x_{1} \\
x_{2}
\end{array}\right)=\lambda_{1}\left(\begin{array}{ll}
3 & 0 \\
0 & 4
\end{array}\right)\left(\begin{array}{l}
x_{1} \\
x_{2}
\end{array}\right)+\lambda_{2}\left(\begin{array}{ll}
5 & 0 \\
0 & 6
\end{array}\right)\left(\begin{array}{l}
x_{1} \\
x_{2}
\end{array}\right) \\
& +\lambda_{3}\left(\begin{array}{ll}
7 & 0 \\
0 & 8
\end{array}\right)\left(\begin{array}{l}
x_{1} \\
x_{2}
\end{array}\right) \\
& \left(\begin{array}{ll}
2 & 0 \\
0 & 1
\end{array}\right)\left(\begin{array}{l}
y_{1} \\
y_{2}
\end{array}\right)=\lambda_{1}\left(\begin{array}{ll}
8 & 0 \\
0 & 9
\end{array}\right)\left(\begin{array}{l}
y_{1} \\
y_{2}
\end{array}\right)+\lambda_{2}\left(\begin{array}{cc}
10 & 0 \\
0 & 15
\end{array}\right)\left(\begin{array}{l}
y_{1} \\
y_{2}
\end{array}\right) \\
& +\lambda_{3}\left(\begin{array}{ll}
14 & 0 \\
0 & 13
\end{array}\right)\left(\begin{array}{l}
y_{1} \\
y_{2}
\end{array}\right) \\
& \left(\begin{array}{ll}
3 & 0 \\
0 & 2
\end{array}\right)\left(\begin{array}{l}
z_{1} \\
z_{2}
\end{array}\right)=\lambda_{1}\left(\begin{array}{ll}
30 & 0 \\
0 & 6
\end{array}\right)\left(\begin{array}{l}
z_{1} \\
z_{2}
\end{array}\right)+\lambda_{2}\left(\begin{array}{cc}
75 & 0 \\
0 & 16
\end{array}\right)\left(\begin{array}{l}
z_{1} \\
z_{2}
\end{array}\right) \\
& +\lambda_{3}\left(\begin{array}{cc}
57 & 0 \\
0 & 14
\end{array}\right)\left(\begin{array}{l}
z_{1} \\
z_{2}
\end{array}\right)
\end{aligned}
$$

To estimate rough bounds of the parameters $\lambda_{1}, \lambda_{2}, \lambda_{3}$ of (3.1.1).(3.1.2),(3.1.3) we go through Gerschgorin theorem. 
Theorem: Let $D_{i}$ be a circle whose centre is $a_{i i}$ and whose radius is $\sum\left|a_{i j}\right|$, where $\mathrm{j}=1,2,3, \ldots, \mathrm{n}$ and $j \neq i$. Then Gerschgorin says that

(1)Every eigenvalue of A must lie in the union of those circles.

(2) If $\mathrm{k}$ of these circles donot touch the other $\mathrm{n}-\mathrm{k}$ circles, then exactly $\mathrm{k}$ eigenvalues lie in the union of those $\mathrm{k}$ circles.

Using (1.2), we can write(3.1),(3.2),(3.3) as

$\operatorname{Diag}(0,0,0,-36,-432,-72,-108,-36) \mathrm{u}=\lambda_{1}$

$\operatorname{diag}(480,84,600,144,264,40,582,152) \mathrm{u}$

Or,

$\operatorname{Diag}(0,0,0,-.2500 .-1.6364,-1.800,-.1856,-.2368) u=\lambda_{1} u$

$\operatorname{Diag}(-72,0,-90,0,-72,-24,-198,-48) \mathrm{u}=\lambda_{2}$

$\operatorname{diag}(480,84,600,144,264,40,582,152) \mathrm{u}$

Or,

$\operatorname{Diag}(-.1500,0,-.1500,0,-.2727,-.6000,-.3402,-.3158) \mathrm{u}=\lambda_{2}$ $\mathrm{u}$

$\operatorname{Diag}(120,12,150,36,336,64,348,92) \mathrm{u}=\lambda_{3}$

$\operatorname{diag}(480,84,600,144,264,40,582,152) \mathrm{u}$

Or,

$\operatorname{Diag}(.2500, .1429, .2500, .2500,1.2727,1.6000, .5979, .6053) \mathrm{u}$ $=\lambda_{3} \mathrm{u}$

Applying Gerschgorin's Theorem we have $\left|\lambda_{1}\right| \leq 0,\left|\lambda_{2}\right| \leq 0,\left|\lambda_{3}\right| \leq 1.6$.

Using these rough bounds of eigenvalues we consider a variety of starting values to calculate the approximate eigenvalues. Eight experiments are displayed in Table.1 and Table 2.

Table:.1

\begin{tabular}{|c|l|l|}
\hline Starting values & $\begin{array}{l}\text { Itera } \\
\text { tion }\end{array}$ & $\lambda^{(\mathrm{k})}$ \\
\hline \multirow{2}{*}{$\lambda^{(0)}=\left(\begin{array}{c}-1 \\
-.15 \\
.3\end{array}\right)$} & 0 & $(-.1,-.15, .3)^{T}$ \\
\cline { 2 - 3 } & 1 & $(-.0329,-.1441, .2597)^{T}$ \\
\cline { 2 - 3 } & 2 & $(-.0167,-.1473, .2552)^{T}$ \\
\cline { 2 - 3 } & 6 & $(-.0004,-.1499, .2501)^{T}$ \\
\cline { 2 - 3 }$\lambda^{(0)}=\left(\begin{array}{c}.1 \\
-.1 \\
.2\end{array}\right)$ & 7 & $(-.0002,-.1500, .2501)^{T}$ \\
\cline { 2 - 4 } & 1 & $(.0559, .0606, .0597)^{T}$ \\
\cline { 2 - 3 } & 3 & $(.0001, .0002, .1427)^{T}$ \\
\hline & & $(1,-.1, .2)^{T}$ \\
\hline
\end{tabular}

\begin{tabular}{|c|c|c|c|}
\hline & & 4 & $(.0000, .0000, .1429)^{T}$ \\
\hline \multirow{4}{*}{$\lambda^{(0)}=$} & \multirow{4}{*}{$\left(\begin{array}{c}-1 \\
-.1 \\
1\end{array}\right)$} & 0 & $(-1,-.1,1)^{T}$ \\
\hline & & 1 & $(-2.2201,-.2160,1.5192)$ \\
\hline & & 2 & $(-1.7635,-.2598,1.3263)$ \\
\hline & & 5 & $(-1.6364,-.2727,1.2727)$ \\
\hline \multirow{5}{*}{$\lambda^{(0)}=$} & \multirow{5}{*}{$\left(\begin{array}{c}-.3 \\
.1 \\
.2\end{array}\right)$} & 0 & $(-.3, .1, .2)^{T}$ \\
\hline & & 1 & $(-.3102, .0337, .2517)^{T}$ \\
\hline & & 2 & $(-.2592, .0051, .2503)^{T}$ \\
\hline & & 3 & $(-.2503, .0002, .2500)^{T}$ \\
\hline & & 4 & $(-.2500, .0000, .2500)^{T}$ \\
\hline \multirow{5}{*}{$\lambda^{(0)}=$} & \multirow{5}{*}{$\left(\begin{array}{c}-.34 \\
-.32 \\
.591\end{array}\right)$} & 0 & $(-.34,-.32, .591)^{T}$ \\
\hline & & 1 & $(-.1401,-.3491, .5844)^{T}$ \\
\hline & & 2 & $(-.1821,-.3409, .5967)^{T}$ \\
\hline & & 3 & $(-.1855,-.3402, .5979)^{T}$ \\
\hline & & 4 & $(-.1856,-.3402, .5979)^{T}$ \\
\hline \multirow{4}{*}{$\lambda^{(0)}=$} & \multirow{4}{*}{$\left(\begin{array}{c}-2.1 \\
-.75 \\
1.8\end{array}\right)$} & 0 & $(-2.1,-.75,1.8)^{T}$ \\
\hline & & 1 & $(-1.8607,-.6267,1.6565)^{T}$ \\
\hline & & 2 & $(-1.8027,-.6011,1.6024)^{T}$ \\
\hline & & 3 & $(-1.8000,-.6000,1.6000)^{T}$ \\
\hline \multirow{6}{*}{$\lambda^{(0)}=$} & \multirow{6}{*}{$\left.\begin{array}{c}-.5 \\
-.5 \\
1\end{array}\right)$} & 0 & $(-.5,-.5,1)^{T}$ \\
\hline & & 1 & $(-.3276,-.3793, .7414)^{T}$ \\
\hline & & 2 & $(-.2566,-.3296, .6348)^{T}$ \\
\hline & & 3 & $(-.2382,-.3168, .6074)^{T}$ \\
\hline & & 4 & $(-.2369,-.3158, .6053)^{T}$ \\
\hline & & 5 & $(-.2368,-.3158, .6053)^{T}$ \\
\hline
\end{tabular}




\section{Table 2}

\begin{tabular}{|c|c|c|c|c|}
\hline Starting & values & $\begin{array}{l}\text { Itera } \\
\text { tion }\end{array}$ & & \\
\hline \multirow{5}{*}{$\lambda^{(0)}=$} & \multirow{5}{*}{$\left(\begin{array}{c}-.1 \\
-.15 \\
.3\end{array}\right)$} & 0 & & .1306 \\
\hline & & 1 & .0785 & .0093 \\
\hline & & 2 & .0171 & .0023 \\
\hline & & 4 & .0022 & $5.4453 \mathrm{e}-004$ \\
\hline & & 7 & $\begin{array}{l}2.8284 \mathrm{e}- \\
004\end{array}$ & $2.8455 \mathrm{e}-004$ \\
\hline \multirow{5}{*}{$\lambda^{(0)}=$} & \multirow{5}{*}{$\left(\begin{array}{c}.1 \\
-.1 \\
.2\end{array}\right)$} & 0 & & .9914 \\
\hline & & 1 & .2178 & .9478 \\
\hline & & 2 & .1150 & .1527 \\
\hline & & 3 & .0108 & .0083 \\
\hline & & 4 & $\begin{array}{l}3.0000 \mathrm{e}- \\
004\end{array}$ & .0031 \\
\hline \multirow{6}{*}{$\lambda^{(0)}=$} & \multirow{6}{*}{$\left(\begin{array}{c}-1 \\
-.1 \\
1\end{array}\right)$} & 0 & & 72.823 \\
\hline & & 1 & 1.3310 & 3.5201 \\
\hline & & 2 & .4976 & .6186 \\
\hline & & 3 & .1292 & .0426 \\
\hline & & 4 & .0093 & .0017 \\
\hline & & 5 & 0 & .0017 \\
\hline \multirow{5}{*}{$\lambda^{(0)}=$} & \multirow{5}{*}{$\left(\begin{array}{c}-.3 \\
.1 \\
.2\end{array}\right)$} & 0 & & 4.1470 \\
\hline & & 1 & .0847 & .9219 \\
\hline & & 2 & .0585 & .1185 \\
\hline & & 3 & .0102 & .0053 \\
\hline & & 4 & $\begin{array}{l}3.6056 \mathrm{e}- \\
004\end{array}$ & 0 \\
\hline \multirow{5}{*}{$\lambda^{(0)}=$} & \multirow{5}{*}{$\left(\begin{array}{c}-.34 \\
-.32 \\
.591\end{array}\right)$} & 0 & & 3.1532 \\
\hline & & 1 & .2021 & .1588 \\
\hline & & 2 & .0445 & .0079 \\
\hline & & 3 & .0037 & $3.0210 \mathrm{e}-004$ \\
\hline & & 4 & $\begin{array}{l}1.0000 \\
\text { e-004 }\end{array}$ & .0012 \\
\hline \multirow{4}{*}{$\lambda^{(0)}=$} & \multirow{4}{*}{$\left(\begin{array}{c}-2.1 \\
-.75 \\
1.8\end{array}\right)$} & 0 & & 28.8110 \\
\hline & & 1 & .3051 & .2344 \\
\hline & & 2 & .0833 & .0067 \\
\hline & & 3 & .0038 & $4.3017 \mathrm{e}-014$ \\
\hline \multirow{4}{*}{$\lambda^{(0)}=$} & \multirow{4}{*}{$\left(\begin{array}{c}-.5 \\
-.5 \\
1\end{array}\right)$} & 0 & & 2.5 \\
\hline & & 1 & .3334 & .5656 \\
\hline & & 3 & .0354 & .0067 \\
\hline & & 5 & $\begin{array}{l}1.0000 \mathrm{e}- \\
004\end{array}$ & .001 \\
\hline
\end{tabular}

The approximate eigenvalues obtained from Table 1 are

$$
\begin{aligned}
& (-.0004,-.1499, .2501)^{T},(0,0, .1429)^{T},(-1.6364,-.2727,1.2727)^{T} \\
& ,(-.25,0, .25)^{T},(-.1855,-.3402, .5979)^{T},(-1.8,-.6,1.6)^{T} \\
& ,(-.2368,-.3158, .6053)^{T}
\end{aligned}
$$

The successive difference between the eigenvalues obtained from table 1 and the successive values of $f(\lambda)$ are calculated in Table2,

\section{CONCLUSION}

Table 2 shows that the successive difference between the eigenvalues are gradually decreases and the values of $f(\lambda)$ also ceases to zero. So the method converses to the exact eigenvalues rapidly. From Table 2 we can say that this method is computationally attractive and we can use this method easily to solve three-parameter eigenvalue.problems. In the process describe above the approximate eigenvalues can be obtained more easily and the convergence is almost guaranteed as well as being more rapid. It will definitely play significant roles for further research in tackling the three-parameter for matrices and the multiparameter problem in general.

\section{REFERENCES}

[1] Atkinson, F.V.,1972. 'Multiparameter Eigenvalue Problems', (Matrices and compact operators) Academic Press, New York, Vol.1

[2] Atkinson, F.V., 1968. 'Multiparameter spectral theory', Bull.Am.Math.Soc., Vol.75, pp(1-28)

[3] Baruah, A.K., 1987. 'Estimation of eigen elements in a two-parameter eigen value problem', Ph.D Thesis, Dibrugarh University, Assam.

[4] Binding, P and Browne P. J., (1989). 'Two parameter eigenvalue problems for matrices', Linear algebra and its application, $p p(139-157)$

[5] Browne, P.J., 1972. 'A multiparameter eigenvalue problem'. J. Math. Analy. And Appl. Vol. 38, pp(553568)

[6] Changmai, J., 2009. 'Study of two-parameter eigenvalue problem in the light of finite element procedure'. Ph. D Thesis, Dibrugarh University, Assam.

[7] Collatz, L.(1968). 'Multiparameter eigenvalue problems in linear product spaces', J. Compu. and Syst.Scie., Vol. 2, pp(333-341)

[8] Devidenko, D.F.(1960). Algorithms for $\lambda$-matrices, Soviet Math., 1, 316-319.

[9] Fox, L., Hayes, L. And Mayers, D.F., 1981. 'The double eigenvalue problems, Topic in Numerical Analysis ', Proc. Roy. Irish Acad. Con., Univ. College, Dublin, 1972, Academic Press, pp(93-112)

[10] Golub, G.H. and Van Loan, C.F. (1983). Matrix Computation, The Johns Hopkins University Press, Baltimore, Maryland.

[11] Horn, R.A,1994. ' Topics in Matrix Analysis'. Cambridge, Cambridge University. 
[12] Hua Dai ${ }^{\text {a }}$ 2007. "Numerical methods for solving multiparameter eigenvalue problems," International Journal of Computer Mathematics, 72:3, 331-347

[13] Konwar, J., 2002. 'Certain studies of two-parameter eigenvalue problems', Ph.D Thesis, Dibrugarh University, Assam.

[14] Lancaster, P. (1966). Lambda-Matrices and Vibrating Systems, Oxford, Pergamon Press.

[15] Plestenjak, B., 2003. Lecture Slides, ' Numerical methods for algebraic two parameter eigenvalue problems', Ljubljana, University of Ljubljans.
[16] Roach, G.F., (1976). 'A Fredholm theory for multiparameter problems', Nieuw Arch. V. Wiskunde, Vol.XXIV(3), pp(49-76)

[17] Sleemen, B. D., 1971. 'Multiparameter eigenvalue problem in ordinary differential equation'. Bul. Inst. Poll. Jassi. Vol. 17, No. 21 pp(51-60)

[18] Sleeman, B.D., 1978, "Multiparameter Spectral Theory in Hilbert Space," Pitman Press, London 